\title{
BIODEGRADATION STUDIES ON POLYETHYLENE DEVELOPED THROUGH PHASE TRANSFER CATALYSED GRAFT COPOLYMERISATION \& RELATED POLYMERS
}

*Inderieet Kaur, *Simrat Kaur and **Ramesh Dogra

\section{Synopsis}

In an attempt to modify polyethylene(PE) with a view to impart biodegradability, graft copolymerisation of vinyl monomers ( $A A m$ \& AAc) onto PE through phase transfer catalysis has been studied. The \%age of grafting of AAm \& AAC onto PE was found to be dependent of $B O P$ cone, monomer cone, time, temperature \& concentration of phase transfer catalyst. Biodegradation of samples of PE-g-poly (AAm) \& PE-g-poly (AAc) prepared by different methods of grafting such as chemical, UV, gamma-radiation induced \& by PTC methods was carried by soil burial tests. It was found that grafted samples prepared by PTC methods showed better results of biodegradation as compared to other.

* Department of Chemistry, Himachal Prodesh University, Shimlo - 171005

** Department of Biofechnology, GGDSD College, Chandigarh. 


\section{Introduction}

Biodegradation term in recent years become a part of green vocabulary. The benefits conferred on society by the development of man made polymers have in recent years observed by the problem of their ultimate disposal. Improperly disposed polymers are significant source of environmental pollution potentially harming wild life $\mathrm{e}^{(1)}$. Most polymers are resistant to attack by micro organisms. Various attempts have been reported to develop biodegradable polymers. To induce biodegradability the polymers need to be modified so that they undergo biodegradation in appropriate waste management infra structure to environmentally compatible constituents (e.g. $\mathrm{CO} 2, \mathrm{H} 2 \mathrm{O}$ or methane \& humus materials leaving no persistent or toxic residues). The following information concerning biodegradability is useful for the synthesis of biodegradable polymers ${ }^{(2)}$;:

1) Ester, ether \& peptide bond are easily degradable; Branching slows degradation.

2) Introduction of $\mathrm{OH} \&$ carbonyl groups accelerate biodegradation:

3) Unsaturation compounds is more biodegradable than corresponding saturated compound.

4) The following trends have been recognized w.r.t. the relative biodegradability of polymers.

i. Aliphatic $>$ Alicyclic $>$ Aromatic polymers,

ii. Hydrophilic polymers $>$ Hydrophobic polymers

iii. Low mol. wt. Polymers $>$ High mol. wt. Polymers

iv. Low m.pt polmers $>$ High m.pt polymers

v. Amorphous polymers $>$ Crystalline polymers

Modification of polymers by graft copolymerisation can add many properties to the trunk polymer. Numerous methods have been suggested for the preparation of graft copolymerisation including chemical ${ }^{(3,4,5)} \&$ radiation techniques. The common feature of most methods is that active site is created in a pre-existing macro molecule. A graft copolymer is then obtained from this activated polymer by polymerization of an appropriate monomer. Use of phase transfer catalyst (PTC) for carrying out the polymerization reaction is a new method used recently for polymerization of some monomers. Tagle efaj ${ }^{(6)}$ carried out the polymerization of 2,2 bi(l,4-phenyleneoxy) propylidene diacetic acid \& related acids with diphenols using several ammonium \& phosphonium salts as phase transfer catalyst. Choi \&. Lee ${ }^{(7)}$ reported that the polymer poly(methyl methacrylate) prepared by phase transfer catalysed polymerization was found to have considerable mol. wt. than polymers prepared by conventional organic initiators. Graft copolymerisation using PTC has not been 
explored. So an attempt has been made to graft copolymerise acrylamide \& acrylic acid onto PE using tetra-butyl ammonium bromide as phase transfer catalyst. Biodegradation studies of PE \& grafted PE by soil burial test to see the effect of introduction of functionalities in PE towards degradability were carried. A plot of \%wt. Loss as a function of \%age of grafting (fig 7) shows that the sample with lower \%age of grafting shows higher wt. loss. Sample of PE grafted with AAc show higher wt. loss as compare to sample of PE grafted with AAm. This is due to the reason that amide function is more stable towards hydrolysis as compare to the $\mathrm{COOH}$ group. Different samples of PE-g-poly AAm) prepared by photochemical, chemical \& gamma radiation induced graft polymerized were subjected to soil burial tests. Loss in wt. was noted after regular intervals.

\section{Material \& Methods}

Commercial polyethylene (PE) in the form of beads were recrystallised from $p$ xylene. Benzoyl peroxide (S.D. fine chemical Pyt $L t d$ ) was used as a free radical initiator, $p$-xylene (Merck) was used as solvent for PE \& BPO. Methanol SD fine chem. Pvt Ltd was used as nonsolvent for precipitation of the back bone polymer, grafted copolymer, polyacrylamide \& polyacrylic acid. Acrylamide (Merck) was dried before use. Acrylic acid. (SD fine chemical Pvt Ltd) was distilled before use. Distilled water was used as a solvent for AAm \& AAC.

\section{Recrystallization of PE}

PE beads were dissolved in $p$-xylene by heating \& filtered. The filtrate was precipitated by addition of methanol. The mixture was filtered again. The residue was washed dried \& crushed to a fine powder.

\section{Graft copolymerization}

Recrystallised PE \& a definite amount of benzoyl peroxide were dissolved in a definite amount of $p$-xylene in a reaction flask. So it was added a definite amount of AAm/AAc dissolved in a known volume of water. Then a definite quantity of PTC (tetrabutyl ammonium bromide) was added to the reaction mixture. The reaction flask was placed in a water bath at-constant temperature under constant stirring for a definite period of time. After the stipulated time period, $\mathrm{CH} 3 \mathrm{OH}$ was added to the reaction mixture to precipitate the grafted copolymer, homopolymer \& the unreacted back bone polymer (PE). The reaction mixture was filtered \& washed thoroughly with hot water to remove the homopolymer. The grafted PE was dried at $45^{\circ} \mathrm{C} \&$ weighed till constant weight was obtained.

$$
\text { \%age of grafting }=\frac{W_{2}-W_{2}}{W_{2}} \times 100
$$


$W_{1} \& W_{2}$ are the weight of original PE \& grafted PE after complete removal of the homopolymer respectively.

\section{Evidence of Grafting}

IR Spectroscopy: On comparison of IR spectra of PE, PE-g-poly (AAm) \& PE-gpoly $(A A c)$, it is observed that the peaks at $3374.5 \mathrm{~cm}^{\prime \prime} \& 3197.8 \mathrm{~cm}^{\prime \prime}$ due to $>N$. $\mathrm{H}$ stretching, at $1674.7 \mathrm{~cm}^{\prime \prime}$ due to amide -1 \& at $1611.4 \mathrm{~cm}^{\prime \prime}$ due to amide -11 in PE-g-poly(AAm) \& the peak in PE-g-poly(AAC) at $1725.2 \mathrm{~cm}^{\prime \prime}$ due to $>C=0$ stretching are absent in unmo'dified PE. This confirmed the formation of graft copolymers.

\section{Biodegradation}

Different samples of varying \%age of grafting of AAm \& AAc prepared by PTC method \& samples of PE -g-poly(AAm) prepared by UV, Gamma induced radiation, chemical methods \& unmodified PE samples were taken in separate tubes containing weighed garden soil samples. The tubes are tightly closed \& kept in the open. The tubes are weighed after regular intervals of time. A reference tube containing only the garden soil was also taken. Anyweight loss in the tube containing garden soil was taken into account while noting the weight of the sample tube containing the grafted samples. The \%age weight loss in all the tubes was plotted against the number of days (fig. 6). Also plot of \%age of weight loss as a function of the \%age of grafting (fig.7) was made.

\section{Result and Discussion}

Dibenzoyl peroxide (BPO) is a known free radical initiator for carrying out the polymerization of various vinyl monomers and graft copolymerization reactions. The general mechanism of the initiating ability of dibenzoyl peroxide is well known and involves the following steps:
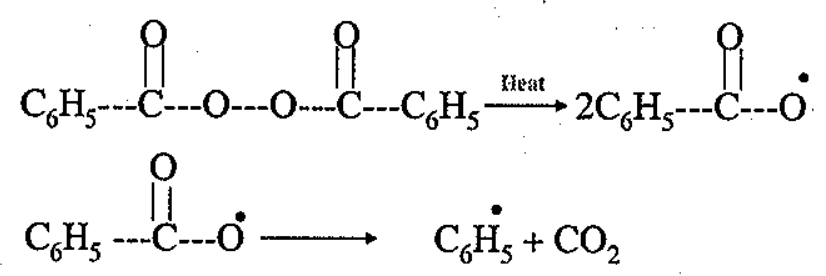
The phenyl radical $(R)$ has the ability to abstract hydrogen atom from the polymeric backbone, polyethylene in the present case, to initiate the copolymerization reaction. The initiation of the monomer is also facilitated by the phenyl radical. The reactions can be predicated as :

$$
\begin{aligned}
& \mathrm{R}^{*} \sim \sim \mathrm{CH}_{2}--\mathrm{CH}_{2}--\mathrm{CH}_{2} \sim \underset{\sim \sim}{\sim} \mathrm{CH}_{2}-\cdots \dot{\mathrm{CH}} \sim-\mathrm{CH}_{2}-\cdots+\mathrm{R}--\mathrm{H} \\
& \mathrm{R}^{*}+\mathrm{M} \longrightarrow \mathrm{R}-\mathrm{M} \stackrel{\mathrm{nM}}{\longrightarrow} \mathrm{R}(\mathrm{M})_{\mathrm{n}}--\mathrm{M}
\end{aligned}
$$

Where $R$ is a radical species generated from BPO. Since the reaction is being carried out in two-phase system, reaction (i) takes place in the organic phase. Reaction (ii) occurs after the transport of the monomer, in the aqueous phase, to the organic phase where it comes in contact with the radical $R$ or with the macroradical (PE).

The interaction of the reactants i.e., PE, monomer, and the initiator distributed between the two phases i.e., the organic and the aqueous phase is due to the presence of phase transfer catalyst i.e., Bu $4 \mathrm{~N}+\mathrm{Br}-$.

The transfer of the reactants from the aqueous phase to the organic phase by PTC can be visualized as follows :

\section{PTC Mechanism}

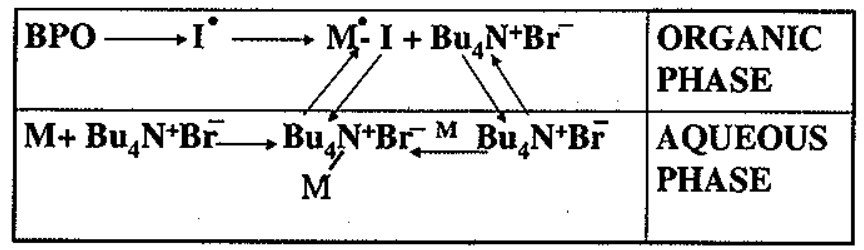

PTC MECHANISM FOR POLYMERIZATION OF MONOMERS

\begin{tabular}{|l|l|l|}
\hline $\mathrm{PE}+\mathrm{BPO} \longrightarrow \mathrm{PE}^{\circ} \longrightarrow \mathrm{PE} \cdot \mathrm{M}^{\circ}+\mathrm{Bu}_{4} \mathrm{~N}^{+} \mathrm{B} \overline{\mathrm{r}}$ & $\begin{array}{l}\text { ORGANIC } \\
\text { PHASE }\end{array}$ \\
\hline $\mathrm{M}+\mathrm{Bu}_{4} \mathrm{~N}+\mathrm{Br} \longrightarrow \mathrm{Bu}_{4} \mathrm{~N}^{+} \mathrm{Br} \stackrel{\mathrm{M} \mathrm{Bu}_{4} \mathrm{~N}^{+} \overline{\mathrm{Br}}}{\longrightarrow}$ & $\begin{array}{l}\text { AQUEOUS } \\
\text { PHASE }\end{array}$ \\
\hline
\end{tabular}

PTC MECHANISM FOR GRAFT COPOLYMERIZATTON OF MONOMERS ONTO PE

PE and BPO being soluble in p-xylene are present in the same phase (organic phase). BPO generates the initiating radicals, which form active PE macrofree radicals by abstracting hydrogen from $P E$ and on this activated $P E$ the grafting will 
take place. The monomers ( $A A m$ or $A A C$ ) being soluble in water are present in aqueous phase. PTC is soluble in both organic as well as aqueous phase, so it transports the water-soluble monomer to the organic layer. The initiation of the monomer in the organic phase takes place by the initiator radical and by the PE macroradical present in the organic layer. The monomer free radical terminates either to produce the graft copolymer or to form the homopolymer. The copolymerization of AAm and AAC on to PE has been studied as a function of different reaction parameters such as Initiator concentration, Monomer concentration, PTC concentration., Time and Temperature. The resulis are explained in the light of the above proposed mechanism.

Optimum \%age of grafting of AAm \& AAC (48\%) onto PE (figl) was observed at $[\mathrm{BPO}]=4.4 \times 10^{12}$ moles \& $8.8 \times 10^{12}$ moles respectively beyond which it decreased. The decrease in grafting beyond optimum [BPO] may be due to:

i) Induced decomposition of $\mathrm{BPO}$ at higher cone.<smiles>O=C(OOC(=O)c1ccccc1)c1ccccc1</smiles>

ii) Homo'polymer formation at the expense of grafting.

Studies on monomer concentration on grafting revealed (fig. 2) that optimum yield was $79 \%$ at $[\mathrm{AAm}]=1.4 \times 10^{12}$ moles beyond which it decreased. Preferential homopolymer as compare to grafting at high monomer concentration will increase viscosity of the aqueous medium. This would result in restricted movement of reactants in aqueous phase \& also the transportation from aqueous phase to organic phase would become difficult which would lead to decrease in percentage of grafting. The effect of [PTC] on graffing gave optimum yield $50 \%$ of AAm \& $68 \%$ of AAC at [PTC] $=3.1 \times 10^{12}$ moles $\& 2.4 \times 10^{12}$ moles (fig. 3 ) respectively. There was decrease in grafting with increase in [PTC] beyond this value. The initial increase grafting with increasing $[P T C]$ is due to the reason that the catalyst helps in transporting the monomer from the aqueous phase to organic phase. Further increase in [PTC] results in increase in transportation of rnonomer to the organic phase leading to more homopolymerization \& decreasing grafting. $V$

Fig.(4) show the effect of time on \%age grafting. Maximum grafting $79 \%$ for AAm \& $91 \%$ for $A A c$ was within 120 minutes \& after this it decreased which may be due to induced decomposition of the initiator. Temperature increased the \%age upto 
$79 \% \mathrm{AAm} \& 91 \%$ for $A A c$ at $60^{\circ} \mathrm{C}$ (fig. 5). Further increase in temperature decreases the \%age of grafting. Previous increase in grafting with rise in temperature was attributed to more free radical formation \& generation of active sites on the polymer backbone. However decrease in grafting after further rise in temp, beyond $60^{\circ} \mathrm{C}$ is attributed to premature termination of growing polymeric chains.

\section{Biodegradation Studies}

The main attacking agent in biodegradation are micro organism e.g. actinomycetes, fungi, bacteria etc. which are wide spread in soil, water \& air. One of the potential approaches to render the synthetic polymers micro organism susceptible would be to incorporate biodegradable structural unit ${ }^{(8>9)}$ into polymeric backbone. In order to find that whether the grafting AAm \& AAc onto PE using phase transfer catalyst has rendered PE as biodegradable or not, biodegradation of the grafted samples was studied as a weight loss in soil burial studies \& the result were compared with that of unmodified PE (which showed almost negligible degradation).

It is observed (fig6) that all the samples of PE grafted AAm \& AAC showed rapid decrease in weight in the early 20 days becomes constant upto 35-37 days; after which a further loss in weight is observed. Maximum weight loss is observed for PEg-poly $(\mathrm{AAm})$ with $50 \%$ grafting where as $\mathrm{PE}$-g-poly $(\mathrm{AAC})$ sample with $10 \%$ grafting showed maximum weight loss. Loss in weight decrease with increase in number of days indicating after the initial weight loss the sumples become almost stable $\&$ are not affected by the environmental condition. The \%age weight loss in first eight days may be due to the minor attack of micro organisms which help in losing some small molecules. The degradation process did not continue to the rest of molecules. This may be due to lack of proper conditions such as optimum water balance, $\mathrm{pH}$ open area required for degradation. A plot of \%age wt. loss as a function of \%age grafting (fig. 7) shows that samples of PE grafted with AAC show higher weight loss as compare to PE grafted with AAm. This is due to the reason that amide function is more stable towards hydrolysis as compare to $-\mathrm{COOH}$ group.

Different samples of PE-g-poly(AAm) already prepared by different methods such as photochemical, chemical \& gamma radiations were also subjected to soil burial tests. Loss in weight was noted after regular intervals. It was observed that the samples prepared by chemical methods, using $\mathrm{CAN} / \mathrm{HNO} 3$ as redox initiator \& gamma radiations methods showed initial loss of $0.92 \% \& 1.03$ days respectively in first 13 days. Gamma radiation samples remained constant for next 43 days while a small weight loss of 0.32 was observed with CAN/HNO3 samples. No change in weight for sample by photochemical methods. From these observations 
it is indicated that the method of synthesis of graft copolymer also plays an important role. Grafted samples prepared by PTC method showed better results as compare to the sample prepared by other methods. The biodegradation followed the order i.e.

PTC method > chemical methods $>$ Gamma radiation method $>$ Photochemical method

In continuation of the synthesis of biodegradable PE through phase transfer catalysed graft coplymerisation of AAm \& AAc. We have synthesized biodegradable natural polymers e.g. starch, dextrin, soya protein, through intercross linking methods. The biodegradable studies with respect to soil burial tests have given very promising results. The \%age wt. loss in soil burial test after 30 days $\& 75$ days for PE \& PE-gcolpolymers are as follows :

\begin{tabular}{llcc}
\hline Sr.No. & Sample & $\begin{array}{c}\text { Degradation after } \\
\text { 30 days }\end{array}$ & $\begin{array}{c}\text { Degradation after } \\
75 \text { days }\end{array}$ \\
\hline 1. & PE-g-starch & $0.94 \%$ & $5.11 \%$ \\
2. & PE-g-soya protein & $1.2 \%$ & $3.87 \%$ \\
3. & PE & $1.52 \%$ & $4.61 \%$ \\
4. & PE-g-dextrin & $4.58 \%$ & $13.49 \%$ \\
\hline
\end{tabular}

Maximum biodegradation has been observed in PE-g-dextrin where as biodegradation of PE-g-soya protein was found to be even less than PE alone. 
Effect of [BPO] on Pg of AAm \& AAC onto PE

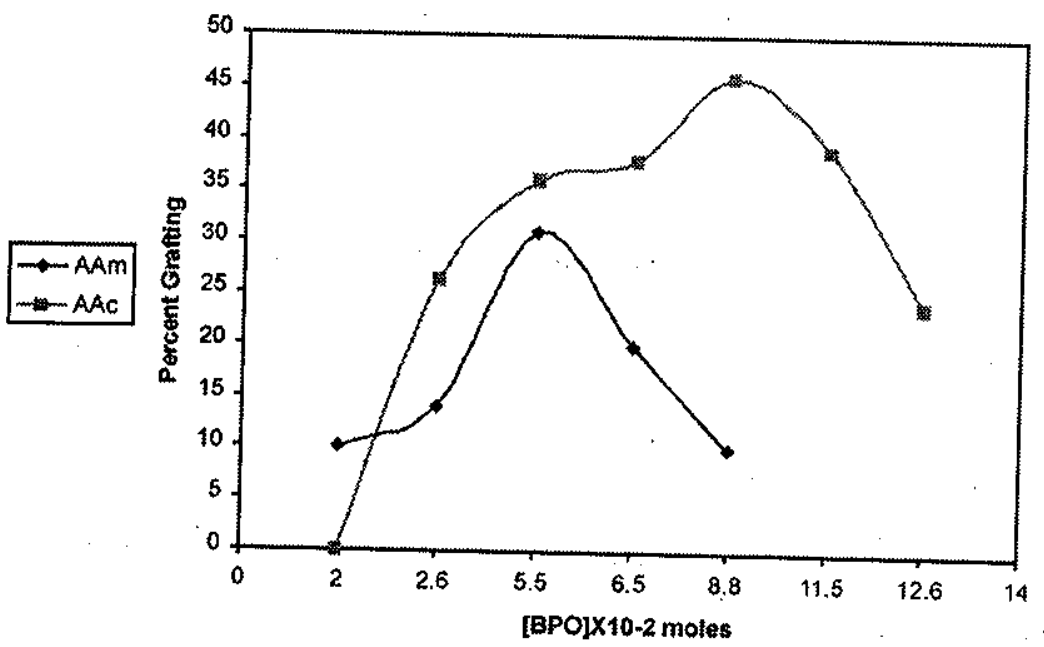

Effect of [M] on Pg of AAm \& AAC onto PE

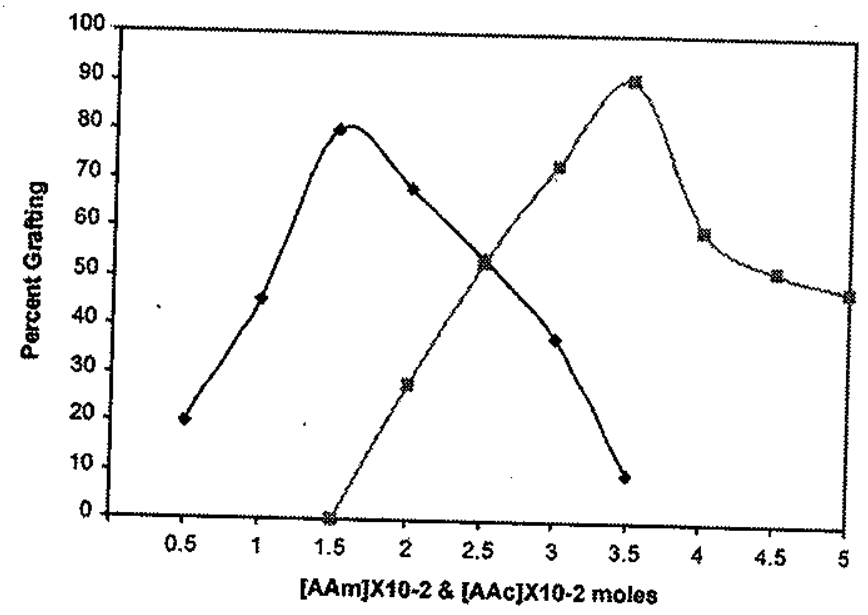


Effect of [PTC] on Pg of AAm \& AAC onto PE

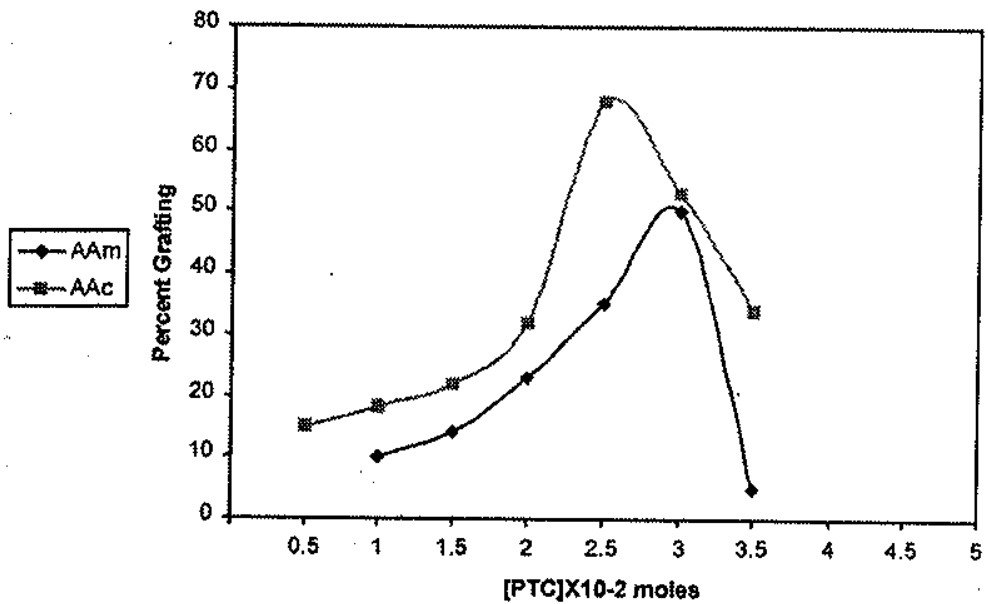

Effect of Variation of time on Pg of AAm \& AAC onto PE

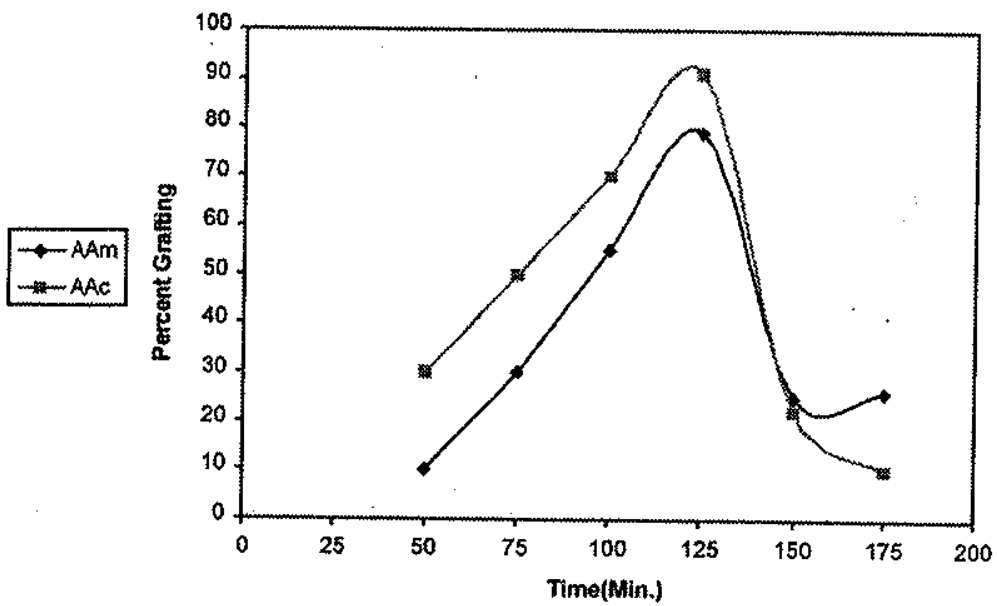


Effect of Varitation of temperature on $\mathrm{Pg}$ of AAm \& AAC onto PE

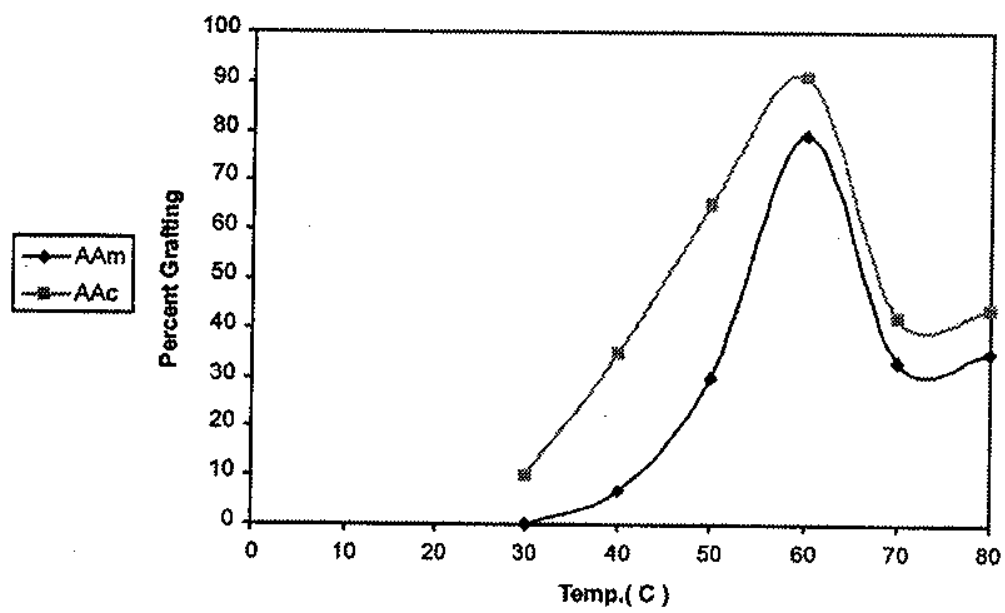

Biodegradation Study of PE-g-copolymers by soil burial test

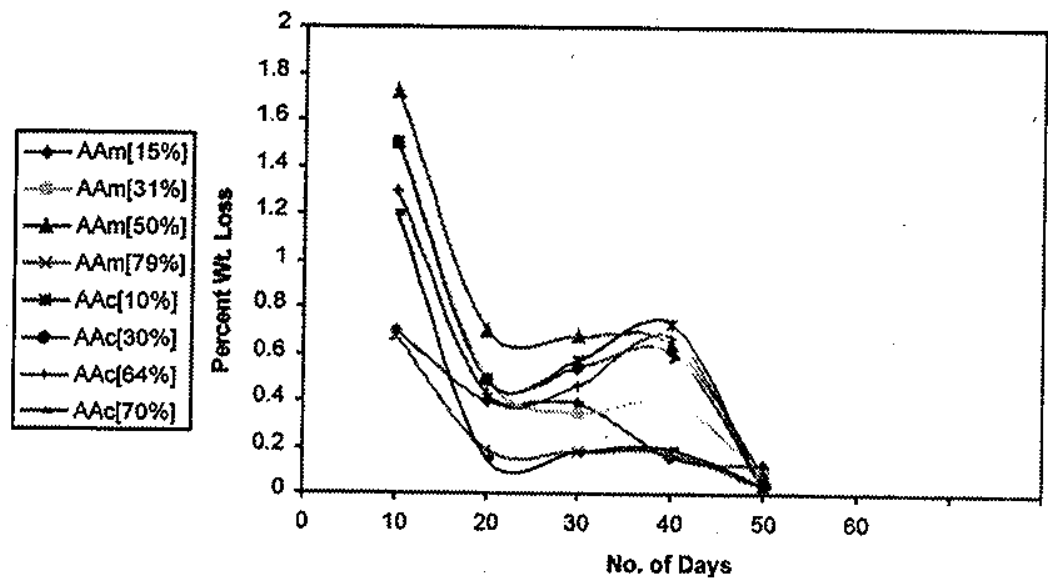


Biodegradation studies of PE-g-Poly(AAm)\& (AAC) Samples

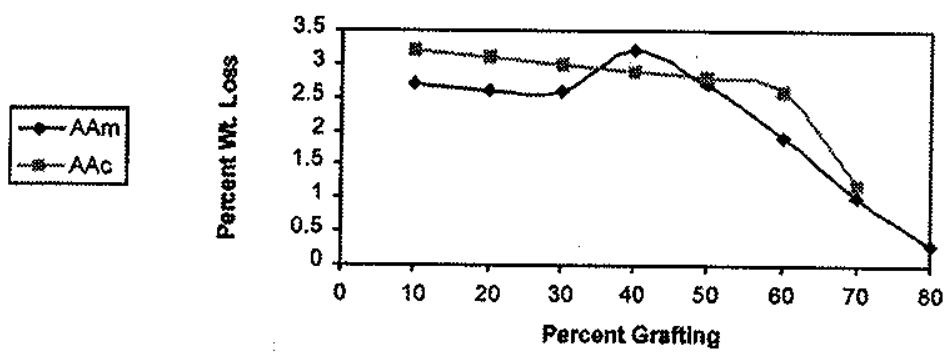

\section{Comparison of biodegradation of PE \& PE grafted with Natural Polymers by soil burial tests}

\begin{tabular}{l}
\multicolumn{2}{c}{ Sample } \\
$\begin{array}{c}\text { Degradation } \\
\text { after 30 days }\end{array}$
\end{tabular} $\begin{gathered}\begin{array}{c}\text { Degradation after } \\
75 \text { days }\end{array} \\
\begin{array}{|l|l|l|l|}\hline 1 & \text { PE-g-dextrin } & 4.58 \% & 13.49 \% \\
\hline 2 & \text { PE } & 1.52 \% & 4.61 \% \\
\hline 3 & \text { PE-g-soyaprotien } & 1.2 \% & 3.87 \% \\
\hline 4 & \text { PE-g-starch } & 0.94 \% & 5.11 \% \\
\hline\end{array}\end{gathered}$

